Results Patients: To date 29 patients have been switched. The median time since switch is 9 months. 25 patients are $>6$ months post switch and included in the analysis. The median age was 61 (range: 28-81) years, 84\% male, 60\% Caucasian, 28\% Black African and $12 \%$ Asian. At LT 6 were acute HBV with Liver failure, 7 had HCC and 4 had delta co-infection. At LT 22 had detectable HBV DNA, 11 were on lamivudine (LAM) and 1 was on LAM and adefovir (ADV). $52 \%$ patients had archived samples suitable for drug resistance testing. None had evidence of any drug resistant mutations.

Results: Since LT all had received HBIG IM with HBsAb levels of 170 (range 101-454) $\mathrm{mIU} / \mathrm{ml}$. 80\% were receiving concurrent LAM, $4 \%$ LAM and ADV and 16\% no oral anti-HBV agent. The median time from LT to switch was 10 (range 2.6-20.3) years. At switch HBsAg and HBV DNA was undetectable in all subjects. $92 \%$ were on calcineurin inhibitor based immunosuppressive regimens. Serum creatinine was 104 (range 62-170) $\mu \mathrm{mol} / \mathrm{l}$, estimated glomerular filtration rate (eGFR) was $65 \mathrm{ml} / \mathrm{min}, 24 \mathrm{~h}$ urine creatinine clearance was 76 (range $41-150) \mathrm{ml} / \mathrm{min}$ and total protein excretion was 81 (range 31-441) mg/day. Serum ALT was $26 \mathrm{IU} / \mathrm{l}$, phosphate was $0.98 \mathrm{mmol} / \mathrm{l}$ and vitamin D was $14 \mu \mathrm{g} / \mathrm{l} .12$ had hypertension and/or diabetes. 16 patients were switched to TDF, 9 patients with eGFR $<60 \mathrm{ml} / \mathrm{min}$ or renal risk factors were switched to ETV. Six months after switch all patients remained HBsAg and HBV DNA undetectable and there was no difference in serum creatinine [103 (TDF 93, ETV 115) $\mu \mathrm{mol} / \mathrm{l}$ ], eGFR [65 (TDF 70, ETV 57) $\mathrm{ml} / \mathrm{min}$ ], ALT (23 IU/l), phosphate $[1.03$ (TDF 1.00, ETV 1.05) $\mathrm{mmol} / \mathrm{l}]$ and vitamin D [17 (TDF 16.3, ETV 18.5) $\mu \mathrm{g} / \mathrm{l}]$. No therapy withdrawal/ change was required due to adverse effects. The approximate drug cost saving made per patient/year from switching from HBIG to TDF or ETV is $£ 11000$ and $£ 10000$ respectively.

Conclusion A stratified conversion protocol, based on the assessment of virological parameters and renal co-morbidities, ensures patients can safely and effectively be switched from HBIG to TDF or ETV to prevent HBV recurrence post LT. HBsAg and HBV DNA remains undetectable and no deterioration in renal function has been observed to date. Significant drug cost savings can be achieved utilising this protocol.

\section{P78 LONG-TERM ANTIBIOTIC PRESCRIPTION IN PATIENTS RELISTED FOR LATE HEPATIC ARTERY THROMBOSIS IS ASSOCIATED WITH GREATER WAITING LIST MORTALITY INDEPENDENT OF MELD}

doi:10.1136/gutjn-2011-300857a.78

M Smith, J Leithead, L Materacki, V Sagar, B Gunson, S Bramhall, D Mutimer, T Shah. Liver Unit, Queen Elizabeth Hospital, Birmingham

Introduction Optimal prioritisation and medical management of patients with late hepatic artery thrombosis (HAT) awaiting liver transplantation remains unclear.

Aim To examine the association of complications of late HAT and their interventions with liver transplant waiting list mortality.

Method Single centre study of 49 patients listed for late HAT 01/ 1995-06/2010. Late HAT was defined as occurrence $>4$ weeks following liver transplantation. Cox regression was adjusted for listing MELD score at all times. Despite increasing waiting time statistical analyses did not demonstrate any influence of listing time period.

Results Mean listing MELD score was 16 (SD 7). 29\% of patients demonstrated biliary stricture/s, 20\% cholangitis and $63 \%$ biloma/ abscess/s. The estimated 3- and 12-month transplant-free survival following listing was $85 \%$ and $53 \%$, respectively. 36 patients were regrafted, with a median time from listing to transplantation of 45 (IOR 13-167) days.
No relationship was demonstrated between the presence of biliary stricture/s $(p=0.984)$, cholangitis $(p=0.770)$ or biloma/ abscess/s ( $p=0.143$ ), and wait-list mortality. Instead, an increasing number of biloma/abscess drain insertions $(\mathrm{p}=0.038)$ and long-term (LT) prescription of antibiotics $(p=0.029)$ were linked with an increased risk of death. Multi-drug resistant bacteria (MDRB) were cultured in bile/blood more frequently in those receiving LT antibiotics ( $44 \%$ vs $8 \%, p=0.004$ ), and MDRB positivity was also a risk factor for waiting list mortality $(\mathrm{p}=0.033)$. On multivariate analysis the only predictor of death was LT antibiotics (MELD, HR 1.23; 95\% CI 1.04 to $1.44, p=0.013$ : antibiotics, HR 24.20; $95 \%$ CI 1.28 to 455.88, $\mathrm{p}=0.033$ ).

Following regraft, LT antibiotics $(p=0.025)$ and MDRB positivity while listed $(p=0.002)$ remained predictors of patient mortality independent of the preoperative MELD score. The estimated 3 - and 12 -month post transplant survival of patients with MDRB positivity was $63 \%$ and $25 \%$, respectively, and for those without $89 \%$ and $86 \%$ (log-rank $\mathrm{p}=0.001$ ).

Conclusion Patients listed for late HAT receiving LT antibiotics are a high risk group who require greater priority for liver transplantation. Our results raise the possibility that by increasing bacterial resistance LT antibiotics may have a detrimental effect on patient survival.

\section{P79 PLASMA SUCCINYLACETONE IS RAISED AFTER LIVER TRANSPLANTATION FOR TYROSINAEMIA TYPE 1 AND IS ASSOCIATED WITH REDUCED PORPHOBILINOGEN SYNTHASE ACTIVITY SUGGESTING IT IS FUNCTIONAL}

doi:10.1136/gutjnl-2011-300857a.79

${ }^{1} \mathrm{D} C$ Bartlett, ${ }^{2} \mathrm{M}$ A Preece, ${ }^{3} \mathrm{E}$ Holme, ${ }^{4} \mathrm{C}$ Lloyd, ${ }^{1} \mathrm{P}$ N Newsome, ${ }^{4} \mathrm{P}$ J McKiernan. ${ }^{1}$ Centre for Liver Research, University of Birmingham and Liver Unit, Queen Elizabeth Hospital Birmingham, UK; ${ }^{2}$ MD Laboratory, Birmingham Children's Hospital, Birmingham, UK; ${ }^{3}$ Department of Clinical Chemistry, Sahlgrenska University Hospital, Gothenburg, Sweden; ${ }^{4}$ Liver Unit, Birmingham Children's Hospital, Birmingham, UK

Introduction Tyrosinaemia type 1 (TT1) is a rare disorder of tyrosine metabolism leading to accumulation of toxic metabolites such as succinylacetone (SA) and a high risk of hepatocellular carcinoma. Children with TT1 traditionally required liver transplantation (OLT) and while the need for this has reduced since the introduction of nitisinone some still go on to require OLT. Circulating SA inhibits the enzyme porphobilinogen $(\mathrm{PBG})$ synthase and its activity can be used as a marker of functional circulating SA. Elevated urinary SA post OLT thought to be due to local production has been reported. Aim This study describes a novel finding of elevated plasma SA following OLT for TT1.

Method A retrospective analysis was performed of patients treated for TT1 at our institution from 1989 to 2010.

Results In patients who received nitisinone prior to OLT mean urinary SA was elevated at presentation $(159.6 \mathrm{mmol} / \mathrm{mol}$ creatinine, ref. range $<1)$ as was plasma $\mathrm{SA}(17.58 \mathrm{~mol} / \mathrm{l}$, ref. range $<0.01)$ but both became undetectable on nitisinone prior to OLT $(p<0.05)$. This was associated with increased mean PBG synthase activity from 0.032 to $0.99 \mathrm{nkat} / \mathrm{g} \mathrm{Hb}$ (ref. range 0.581 .25 ). In patients who did not receive nitisinone, mean urinary SA was $274.6 \mathrm{mmol} / \mathrm{mol}$ creatinine immediately prior to transplant. Plasma SA levels/PBG synthase activity prior to OLT were not available in this group. Following OLT in patients treated with nitisinone, mean urinary SA levels quickly rose and remained elevated for the duration of follow-up. Plasma SA levels also rose with a progressive decrease in mean PBG synthase activity to low-normal levels. In patients who had not received nitisinone, mean urinary SA fell quickly by 1 year post OLT but remained above normal at levels similar to those seen in the nitisinone treated group. No data were available for plasma SA/PBG synthase activity in this group until 
13 years post OLT. However, from this point onwards mean plasma SA remained elevated with a low-normal PBG synthase activity similar to that seen in nitisinone treated patients. There was no apparent correlation between SA levels and renal function. Despite reduced $\mathrm{PBG}$ synthase levels, no patients developed porphyria-like symptoms.

Conclusion Urinary and plasma SA levels are elevated following OLT for TT1. Low-normal PBG synthase activity suggests the circulating SA may be functional. The clinical significance of this is unclear.

\section{P80 PATIENT CHARACTERISTICS AND OUTCOMES IN A 'HUB AND SPOKE MODEL' FOR LIVER TRANSPLANTATION PROVISION: THE SOUTH WEST LIVER UNIT/KING'S COLLEGE EXPERIENCE}

doi:10.1136/gutjnl-2011-300857a.80

${ }^{1}$ M Petrova, M Fung, M E Cramp, J D Mitchell, T J S Cross. ${ }^{1}$ The South West Liver Unit, Derriford Hospital, Plymouth, Devon, UK

Introduction Liver transplantation (LTx) is the only curative therapeutic modality for patients with end-stage liver disease (ESLD). A detailed evaluation of the liver transplant patient is critical to identify patients most likely to benefit from LTx in an era of organ donor shortage to optimise use of a scarce resource.

Aim The aim of this study was to analyse the profile and outcome of LTx referrals in a 'hub and spoke' LTx service.

Method A retrospective study of all patients referred to the South West Liver Unit, liver transplant service between April 2007 and April 2011. Patients with acute liver failure were excluded from the analysis. Pre-transplant and post-operative follow-up was performed at the South West Liver Unit. All operations were performed at King's College Hospital, London. Pre-LTx demographic and laboratory data were analysed using descriptive methods. Comparisons (Mann-Whitney) and survival (Kaplan-Meier) were estimated. $\alpha$ level of 0.05 was accepted as significant.

Results 191 consecutive patients ( $n=128,67 \%$ males) underwent elective pretransplant assessment and posttransplant management. Mean age was 53 years, SD 10.3 (range 19-70). Currently, 9.4\% (18/ 191) patients are under assessment, $10.5 \%(20 / 191)$ are on the waiting list for LTx, 29.8\% (57/191) have been transplanted, $7.9 \%$ $(15 / 191)$ died on the list and $42.4 \%(81 / 191)$ were assessed but not listed (too advanced disease in $12 \%$, not fulfilling minimal listing criteria $14.7 \%$ and contraindicated $15.7 \%$ ). Among patients who met minimal listing indications $21 \%(n=19)$ were diagnosed with

\section{Survival of all assessed patients}

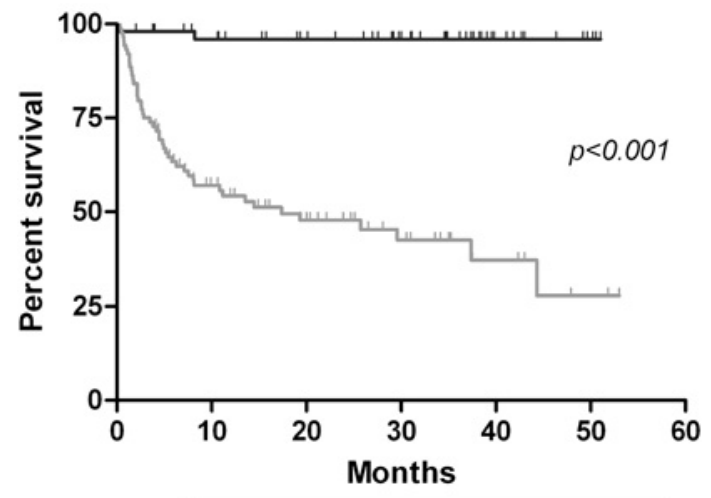

$\leftarrow$ LTX patients $\longrightarrow$ Not transplanted

Abstract P80 Figure 1 Survival of all patients. hepatocellular carcinoma (chronic HCV infection or/and alcohol background), 30\% ( $n=28)$ had alcoholic liver disease, 9\% $(n=8)-$ chronic HCV infection, $11 \%(\mathrm{n}=10)$ exhibit both alcohol and viral aetiology, 4\% ( $n=4)$-autoimmune hepatitis, 11\% ( $n=10)$ - PBC/ PSC, $4 \%$ NASH $(n=4), 2 \%(n=2)$ cryptogenic cirrhosis and in $9 \%$ $(n=8)$ rare diseases (vascular, metabolic, congenital or chronic rejection). These proportions did not deviate from the whole assessed cohort. Mean UKELD, MELD and CTP scores of all assessed patients were 52 (SD 5.2), 12 (SD 5.6) and 8 (SD 1.9). UKELD correlated strongly with MELD and CTP (Spearman's $\rho 0.68$ and $0.72, \mathrm{p}<0.01)$ and was slightly higher in listed for LTx group. CTP score did not differ between transplanted and not transplanted patients. Among the liver recipients $36 \%$ were blood group A, $12 \%$ $\mathrm{B}, 10 \% \mathrm{AB}$ and $42 \% \mathrm{O}$, similar to the distribution in the whole group. Mean BMI was 26.2, not different between transplanted and not listed patients. However, a third of all assessed patients had severe protein malnutrition, evaluated with hand dynamometry and estimated energy expenditure/intake ratio. The prevalence of HPS and $\mathrm{PPH}$ were $9 \%(11 / 122)$ and $3 \%(4 / 135)$ respectively. Three months-, 1- and 3-year survival of the patients and the grafts were $98 \% / 97 \% / 97 \%$ and $98 \% / 95 \% / 90 \%$. Abstract P80 figure 1 illustrates the differences in survival of transplanted and not transplanted patients.

Conclusion Graft and patient survival in the 'hub and spoke' model is good. Alcohol and hepatoma are the commonest reasons for listing. Protein malnutrition is common in this patient cohort suggesting improved patient nutrition and early dietician involvement is needed.

\section{P81 PRE-TRANSPLANT HISTOLOGICAL ASSESSMENT IN PATIENTS WITH ALCOHOLIC LIVER DISEASE DOES NOT PREDICT RISK OF RECIDIVISM POST LIVER TRANSPLANTATION}

doi:10.1136/gutjnl-2011-300857a.81

${ }^{1} \mathrm{~J}$ Prentis, ${ }^{2} \mathrm{~S}$ McPherson, ${ }^{3} \mathrm{~A}$ Burt, ${ }^{2} \mathrm{D}$ Manas, ${ }^{1} \mathrm{C}$ Snowden, ${ }^{2} \mathrm{M}$ Hudson, ${ }^{2} \mathrm{~S}$ Masson. ${ }^{1}$ Department of perioperative and critical care medicine, Freeman Hospital; ${ }^{2}$ Liver transplant Unit, Freeman Hospital; ${ }^{3}$ Institute of cellular medicine, Newcastle University

Introduction Liver transplantation for alcoholic liver disease (ALD) is well established, but it remains a controversial indication. Assessment of patients in whom alcohol has been a contributing factor to chronic liver disease aims to identify those at risk of relapse. As well as psychological assessment, histological evidence of steatohepatitis may indicate ongoing alcohol consumption.

Aim To determine the prevalence of histological evidence of ongoing alcohol use among patients undergoing liver transplant assessment for ALD and its affect on listing and risk of recidivism.

Method Consecutive patients with ALD assessed for liver transplantation between 2006 and 2010 were included. Transjugular liver biopsy was performed routinely in all patients and specimens were reviewed by an expert hepatopathologist. Outcomes including decision to list for transplant, survival and recidivism (patient admission or blood alcohol detection) were recorded

Results 122 patients were included (70\% male, median age 55) with diagnoses of lone ALD (92), ALD/HCV (10) and HCC (10) Histology was insufficient for analysis in four. Most patients (108, $89 \%$ ) had histological evidence of micronodular cirrhosis without evidence of steatosis or steatohepatitis, suggesting abstinence. Of these, 52 were listed for transplantation though two were subsequently removed for recidivism. To date, 39 have been transplanted; 1 -year follow-up was available in 28. The overall 1-year survival was $90 \%$ and $28 \%$ had returned to drinking alcohol $(n=7)$, although none were 'problem' drinkers. 